\title{
Stability of Diodes with Poly(3-hexylthiophene) and Polyazomethines Thin Organic Layer
}

\author{
H. Bednarski ${ }^{a, *}$,J. Gąsiorowski ${ }^{b}$, M. Domański ${ }^{a}$, B. Hajduk ${ }^{a}$, J. Jurusik ${ }^{a}$, \\ B. JARZĄBEK ${ }^{a}$ AND J. WESZKA ${ }^{a}$ \\ ${ }^{a}$ Centre of Polymer and Carbon Materials, Polish Academy of Sciences \\ M. Curie-Skłodowskiej 34, 41-819 Zabrze, Poland \\ ${ }^{b}$ Linz Institute for Organic Solar Cells (LIOS), Physical Chemistry, Johannes Kepler University \\ Altenberger Str. 69, Linz 4040, Austria
}

\begin{abstract}
Herein we report results of studies on stability of diodes based on organic semiconductors such as poly (3-hexylthiophene) (P3HT) and soluble derivative of polyazomethine poly(1,4-(2,5-bisoctyloxy phenylenemethylidynenitrilo)-1,4-phenylenenitrilomethylidyne), (BOO-PPI). Both polymers were deposited on glass/ITO substrate with or without covering with blocking layer: poly(3,4-ethylenedioxythiophene):poly(4-styrene sulfonate) (PEDOT:PSS) and finished with Al back electrode. Prepared devices were studied by monitoring their electrical conductivity under nitrogen atmosphere and ambient air conditions. Under nitrogen atmosphere a marked influence of presence of the blocking layer on the diodes electrical conductivity was revealed. The P3HT diodes prepared without PEDOT:PSS thin film shown quick degradation, whereas presence of these layers stabilizes electrical conductivity in these devices. Inversely, the PPI based diodes without the PEDOT:PSS revealed stable conducting properties, while corresponding diodes with PEDOT:PSS layer showed degradation traces of their conducting properties.
\end{abstract}

PACS: 73.40.Ei, 73.50.Pz

\section{Introduction}

The optoelectronic devices based on organic semiconductor thin films have been recently extensively studied due to their possible low cost production, mechanical flexibility, and easiness of their preparation [1-3]. Much attention was put to experimental and theoretical studies of transport properties of organic semiconductors leading to marked increase in understanding mechanisms which play the key role in operational performance of i.e. photovoltaic devices [1-8]. Research done on poly (3-hexylthiophene) (P3HT), the model material for organic solar cells, provided the substantial knowledge on such phenomena as current limiting mechanisms in ITO/ $\mathrm{P} 3 \mathrm{HT} / \mathrm{Al}$ devices [9], the trap filling and the trap free transport properties [10] as well as on influence of various parameters on overall device performances [11-13].

Practical application in the optoelectronic devices of organic semiconductors is limited by very low stability under normal atmospheric conditions. Degradation processes in air, due to the diffusion of the atmospheric gases into organic layer, as well as oxidation of metal contacts, affect conductivity in organic semiconductors and

* corresponding author; e-mail: hbednarski@cmpw-pan.edu.pl are leading to decrease electrical parameters and overall device efficiency. Up to date influence of air on organic semiconductors stability has been intensively investigated [2, 4-8]. Additionally influence of metal-organic semiconductor interface on cell performance was studied by adding in the cell construction additional PEDOT:PSS layer. In this work we report results of stability study on organic diodes based on organic semiconductors such as P3HT, model material used as donor in organic solar cells, and derivative of the polyazomethine polymer, BOO-PPI. All devices prepared with and without PEDOT:PSS layer between indium tin oxide (ITO) and organic semiconductor, were studied under nitrogen and air atmosphere, by monitoring their electrical, optical and morphological properties, as function of time, using current-voltage $(I-V)$ characteristics, UV-Vis spectroscopy and atomic force microscopy (AFM) imaging.

\section{Polymer thin films preparation and experimental details}

As a front electrode glas/ITO substrates $(15 \Omega / \square$, Kintec Co.) pre-cleaned by subsequential ultrasonic washing with an acetone (10 $\mathrm{min})$, isopropanol (20 min), and deionized water (10 min) were used. After cleaning ITO surface was dried with nitrogen. Part of the studied diode structures were prepared on the cleaned glass/ITO 
substrate covered with PEDOT:PSS (Baytron P, Clevios Co.) interfacial layer. This interfacial layer was spin coated from $70 \%$ solution of PEDOT:PSS diluted in deionised water and dried in air on the hot plate at $150^{\circ} \mathrm{C}$ for $5 \mathrm{~min}$. In our study, soluble derivative of polythiophenes, regioregular poly (3-hexylthiophene) (98\%, Rieke Metals) was used without further purification. The P3HT was dissolved in chlorobenzene (99+\%, Across Organics) with a concentration of $10 \mathrm{mg} / \mathrm{mL}$ and BOO-PPI was dissolved in chloroform $(99+\%$, SigmaAldrich) with a concentration of $6 \mathrm{mg} / \mathrm{mL}$. At a later stage both polymers were deposited by spin coating on the glass/ITO and the glass/ITO/PEDOT:PSS substrates.

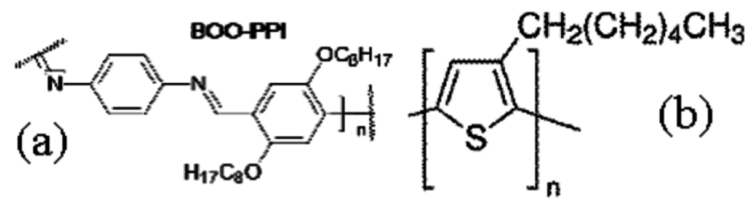

Fig. 1. Polymer repeating units: (a) BOO-PPI, (b) P3HT.

Structures of the polymers described used in this research are presented in Fig. 1 for the BOO-PPI (a) and the P3HT (b), respectively. For studying electrical properties in the diode architecture a $100 \mathrm{~nm}$ thick $\mathrm{Al}$ electrode was evaporated on the top of organic layer. These structures were kept and characterized in a glove-box under nitrogen atmosphere (Linz) or exposed in air (Zabrze). In this manner, we compared the stability of the diodes prepared in two different laboratories starting from the same organic semiconductor basic materials and we had a possibility to evaluate reproducibility as well as quality of particular technological processes connected with the devices fabrication, e.g. deposition of organic thin film or metal electrode. It should be stressed that stability of the organic layered structures without top metallic electrode were monitored using UV-Vis JASCO V-570 spectrometer and Topmetrix AFM analysis, which however have not revealed any visible changes in the position of optical absorption band maxima of the UV-Vis spectra with time. However, we have observed small changes (variations) in the absorbance level.

\section{Results}

Representative current-voltage characteristics taken on ITO/X/BOO-PPI/Al and ITO/X/P3HT/Al (where $\mathrm{X}$ represent lack or existence of PEDOT:PSS layer) diodes under nitrogen gas atmosphere are compared in Fig. 2 and Fig. 3, respectively. Numerical labels on the curves indicate the time in days. The $I-V$ characteristics labeled by 1 were taken on the fresh diodes. As can be seen, the BOO-PPI based diodes revealed relatively stable electrical conductivity, irrespective of presence of interfacial PEDOT:PSS layer. P3HT based diodes without PEDOT:PSS layer showed a quick decrease of their electrical conductivity, within first day, and then stabilized. It is important to note also significantly higher currents in BOO-PPI diodes without PEDOT:PSS layer on the ITO electrode.

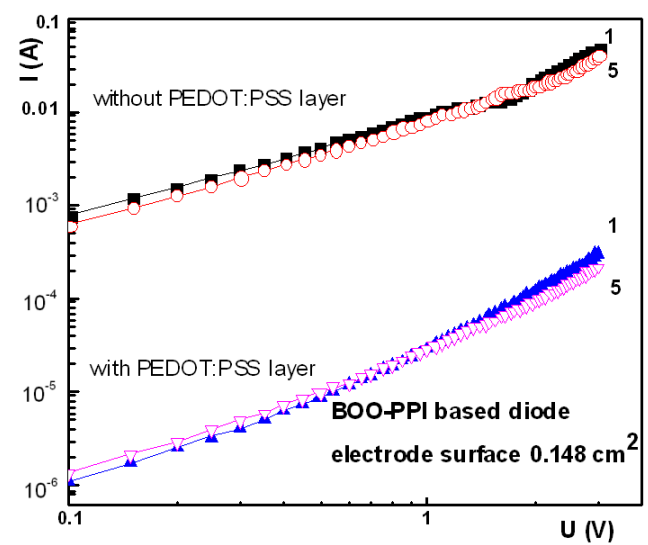

Fig. 2. Comparison of the $I-V$ characteristics for the ITO/PEDOT:PSS/BOO-PPI/Al and ITO/BOO-PPI/Al diodes as measured on fresh devices (marked by 1 ) and after 5 days (marked by 5 ). The devices were kept in nitrogen gas atmosphere.

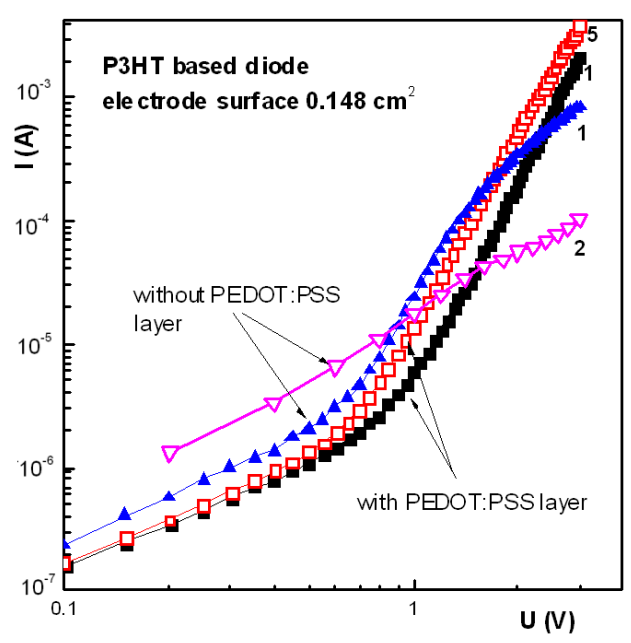

Fig. 3. Comparison of the $I-V$ characteristics for ITO/PEDOT:PSS/P3HT/Al and ITO/P3HT/Al diodes as measured on fresh devices (marked by 1) and after $n$ days (number of days marked on the plots). The devices were kept in nitrogen gas atmosphere.

To carry out studies under ambient air condition, similar structures based on P3HT and BOO-PPI from the same organic materials have been prepared in Zabrze. All the studied structures, without or with PEDOT:PSS interfacial layer, revealed quick degradation of their electrical properties. Practically, within few days we have observed total disappearance of their electrical conductivity. For instance, in Fig. 4 we show the $I-V$ characteristics for ITO/PEDOT:PSS/BOO-PPI/Al device measured in the dark immediately after preparation and after 


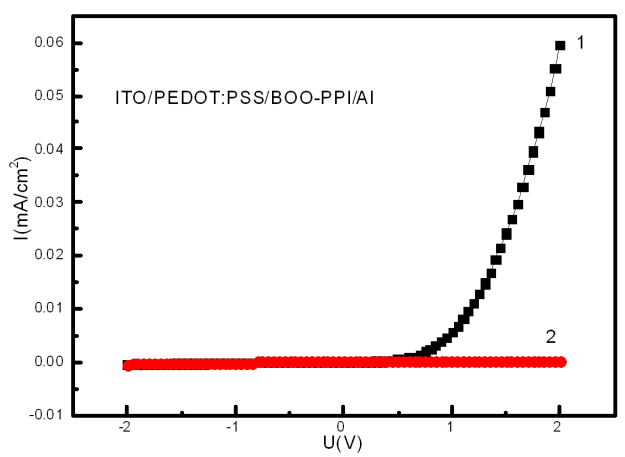

Fig. 4. The $I-V$ characteristics for ITO/PEDOT:PSS/BOO-PPI/Al diodes as measured on fresh devices (marked by 1 ) and one day after (2). The devices were kept in ambient air.

$24 \mathrm{~h}$. One can observe a very quick complete disappearance of the electrical conduction in this case.

\section{Discussion}

To identify different electrical transport mechanisms, in the $\log -\log$ scale, we fitted the experimental forward-dark current with the straight lines, corresponding to the currents with the power law voltage dependence $I \sim V^{n}$ where $n=1,2$ and 5 . The linear and square voltage dependence can be attributed to the ohmic and the trap free space-charge limited conduction (SCLC), while $I \sim V^{5}$ indicates that either trapping or effect of field on mobility is important [14]. Such analysis applied to data presented in Fig. 3 (P3HT based diodes) revealed that the relatively large current $I \sim V^{5}$ disappeared within first day after the device preparation. Then, persisted currents follows Ohm's law voltage dependence and SCLC law with $I \sim V^{2}$. Similar analysis performed on the data presented in Fig. 2 showed that in BOO-PPI based diodes, the linear $V$ dependence directly changes to the trap free SCLC $\left(I \sim V^{2}\right)$ form. This finding allows for the quantitative analyses according to Refs. [9-14].

Most of the organic semiconductors due to the lack of air stability have a natural $p$-type doping in the range $10^{15}-10^{17} \mathrm{~cm}^{-3}$ and reveal ohmic conduction at low voltage (between the ohmic contacts) $[9,14]$ :

$$
J=q n \mu \frac{V}{L}
$$

where $q$ is the charge, $n$ - the charge carrier density, $\mu$ - the carrier mobility, $V$ - the applied voltage and $L-$ the thickness of the organic material. For higher voltage conduction changes to the trap free SCLC $[9,14]$ :

$$
J_{\mathrm{SCLC}}=\frac{9}{8} \varepsilon \varepsilon_{0} \mu \frac{V^{2}}{L^{3}},
$$

where $\varepsilon$ is the dielectric constant of the organic material and $\varepsilon_{0}$ is the vacuum permittivity. When a transition from the Ohm law to the SCLC occurs, the both currents are equal at the transition voltage $V_{\mathrm{tr}}$, which can be determined experimentally. In this case, the doping den- sity $n$ can be calculated from the following relation [14]:

$$
n=\frac{9 \varepsilon \varepsilon_{0} V_{\mathrm{tr}}}{8 q L^{2}} .
$$

The last equation allows us to determine the charge carrier density $n$ from data presented in Fig. 1. In order to calculate corresponding densities, the thickness of the BOO-PPI layer in the diodes $L=160 \pm 10 \mathrm{~nm}$ were measured using the AFM. Determined values of $n$ on fresh diodes for ITO/BOO-PPI/Al (ITO/PEDOT:PSS/BOO$-\mathrm{PPI} / \mathrm{Al})$ are $1.1 \times 10^{16}\left(3.1 \times 10^{15}\right)$ and after four days $1.5 \times 10^{16}\left(5.3 \times 10^{15}\right) \mathrm{cm}^{-3}$, respectively. Let us note, about one order of magnitude higher $n$ in diodes without PEDOT:PSS layer and visible increase of $n$ within the time. This result is attributed to an active role of ITO electrode in increasing doping of BOO-PPI. In this manner, we can regard on the PEDOT:PSS layer as on the buffer layer which prevent the doping by ITO.

We now turn to discuss the results presented in Fig. 2 in more detail. One can observe that $I-V$ characteristics for ITO/P3HT/Al device quickly changed with the time and became qualitatively similar to that presented in Fig. 1, whereas $I-V$ dependences for ITO/PEDOT:PSS/ $\mathrm{P} 3 \mathrm{HT} / \mathrm{Al}$ diodes behave differently. They are stable in time and do not reveal the SCLC in the range of applied voltages. Moreover, one can observe even an improvement of their conduction properties with time (compare corresponding plots 1 and 5), this feature can be attributed to the relaxation processes which increase spatial ordering of the P3HT layer. Nevertheless, the marked importance of the PEDOT:PSS layer in the P3HT based devices is clear. According to the mentioned similarity between the both figures, we can extend our interpretation on the P3HT based devices. Namely, we may also ascribe observed increase in the ohmic current at low voltage in glass/ITO/P3HT/Al device as to caused by the extra doping introduced by the ITO. Inversely, presence of the PEDOT:PSS layer prevents the doping in glass/ITO/PEDOT:PSS/P3HT/Al diodes.

\section{Conclusions}

The studies of stability of P3HT and BOO-PPI based diodes performed under nitrogen atmosphere, clearly indicate an important role of the PEDOT:PSS thin layer on short time stability of electrical conduction in studied diodes. Studies performed on the BOO-PPI based diodes revealed that irrespectively of the presence of the PEDOT:PSS thin layer their forward electronic conduction is dominated by the Ohm law type current at low voltage and by the trap free SCLC with current proportional to $V^{2}$ at higher voltage. The value of the doping density $n$ depends strongly on the presence of the PEDOT:PPS thin layer in the diodes architecture. We have presented convincing arguments that the PEDOT:PSS layer appears as the buffer layer which prevents the doping by the ITO substrate. Moreover, this interpretation is consisting also with our results obtained on the P3HT based diodes. Namely, we can ascribe observed in the time 
increase in the ohmic current of ITO/P3HT/Al diodes as the result of doping introduced from ITO to interfacial P3HT, whereas in the diodes ITO/PEDOT:PSS $\mathrm{P} 3 \mathrm{HT} / \mathrm{Al}$ presence of the thin PEDOT:PSS buffer layer prevents the extra doping, simultaneously stabilizing conduction. Performed studies on similar devices kept and measured in ambient air indicated their very low electrical conduction stability which undergoes fast and strong degradation.

\section{Acknowledgments}

We wish to thank Professor Sariciftci, this research could not have been done without his personal commitment and help. The research at LIOS has had financial support from WTZ program no. 8508/R11/R12. The research carried out in the CPCM PAS in Zabrze has been performed in the frame of the project no N N508626840 financed by the National Science Centre.

\section{References}

[1] A.J. Heeger, N.S. Saricftci, E.B. Namds, Semiconducting and Metallic Polymers, Oxford University Press, Oxford 2010.

[2] T. Jeranko, H. Tributsch, N.S. Sariciftci, J.C. Hummelen, Solar Energy Mater. Solar Cells 83, 247 (2004).

[3] P.A. Lane, Z.H. Kafafi, in: Organic Photovoltaics: Mechanisms, Materials and Devices, Ed. S.-S. Sun, N.S. Sariciftci, CRC Press Taylor \& Francis Group, Boca Raton (FL) 2005, p. 49.
[4] F.C. Krebs, K. Norrman, Prog. Photovolt. Res. Appl. 15, 697 (2007)

[5] M. Wang, F. Xie, W. Xie, S. Zheng, N. Ke, J. Chen, N. Zhao, J.B. Xu, Appl. Phys. Lett. 98, 183304 (2011).

[6] Rashmi, A.K. Kapoor, S. Annapoorni, V. Kumar, Semicond. Sci. Technol. 23, 035008 (2008).

[7] Rashmi, A.K. Kapoor, U. Kumar, V.R. Balakrishnan, P.K. Basu, Pramana - J. Phys. 68, 489 (2007).

[8] A. Guerrero, P.P. Boix, L.S. Marchesi, T. Ripolles-Sanchis, E.C. Pereira, G. Garcia-Belmonte, Solar Energy Mater. Solar Cells 100, 185 (2012).

[9] Z. Chiguvare, J. Parisi, V. Dyakonov, J. Appl. Phys. 90, 2440 (2003); Lett. 98, 183304 (2011).

[10] Z. Chiguvare, V. Dyakonov, Phys. Rev. B 70, 235207 (2004).

[11] M. Mingebach, C. Deibel, V. Dyakonov, Phys. Rev. B 84, 153201 (2011).

[12] T. Strobel, C. Deibel, V. Dyakonov, Phys. Rev. Lett. 105, $266602(2010)$.

[13] M. Limpinsel, A. Wagenpfahl, M. Mingebach, C. Deibel, V. Dyakonov, Phys. Rev. 81, 085203 (2010).

[14] S.C. Jain, W. Geens, A. Mehra, V. Kumar, T. Aernouts, J. Poortmans, R. Mertens, M. Willander, J. Appl. Phys. 89, 3804 (2001). 